\title{
Is Quantum Space a Random Cantor Set with a Golden Mean Dimension at the Core?
}

\author{
M. S. EL NASCHIE*
}

\begin{abstract}
"Vielleicht weise der Erfolg von Heisenbergs Methode ... auf die Ausschaltung kontinuierlicher Funktionen aus der Physik hin. Dann aber muss auch auf die Verwendung des Raum-Zeit Kontinum prinzipiell verzichtet werden"' (A. Einstein).
\end{abstract}

In ref. [1], Mauldin et al. proved a theorem which at first sight may seem slightly paradoxical but we perceive as exceedingly interesting. This theorem states that the Hausdorff dimension $d_{c}^{(0)}$ of a randomly constructed Cantor set is $d_{c}^{(0)}=\phi$, where $\phi=(\sqrt{ } 5-1) / 2$ is the Golden Mean. That such disordered indeterministic construction which actually epitomize dissonance should single out the Golden Mean, an epitomy of perfect order and internal harmony, as a Hausdorff dimension strikes us at least in the first instance as suprising. Now if we could extrapolate the random construction of the said Cantor set to $n$ dimensions, then one could utilize some of the results of our recent work on $n$ dimensional Cantor sets [2,3] and claim that the Hausdorff dimension of a four-dimensional version is $d_{c}^{(4)}=(1 / \phi)^{3}$. This is a direct application of the bijection formula $d_{c}^{(n)}=\left(1 / d_{c}^{(0)}\right)^{n-1}$ introduced in refs [2-5]. In other words, by lifting $d_{c}^{(0)}=\phi$ to four dimensions one finds that

$$
d_{c}^{4}=(1 / \phi)^{3}=4.236067977=\sqrt{ } 5+2=4+\phi^{3}=4+d_{c}^{(-2)} .
$$

It is the aim of this short note to glance at the implications of the preceeding construction to the geometry of micro space-time of quantum mechanics.

More precisely, we mean the implications to the comprehensive effort of Nottale [4] to give up differentiability and assume micro space-time to be a fractal and our own slightly more radical proposal to give up even continuity and regard micro space-time as a multidimensional Cantor set [5]. This proposal seems attractive to us for many reasons, but one of the most important is the natural dependence on the resolution which such spaces possess. In other words, the scale covariance of Cantorian geometry [3-8]. This feature is, of course, shared by Nottale's work [4]. Our preference for the "discretum", however, stem from the failure to prove in mathematics the continuum hypothesis. This alone-and apart from the hard experimental evidence of quantum mechanics-should have evicted continuity from physics long ago.

The attraction of a random Cantor set with a Golden Mean Hausdorff dimension as model for micro space-time is also varied. A particularly subtle one is that it solves the problem of transition from a flat very orderly space to a rugged random space governed by probability. Such random space, one must ponder, should inherit some of the order of the 
classical macro space. A definite and also unlikely value for the dimension such as $\phi$ could be viewed as reflecting the orderly origin of this random micro space. A second point is the Golden Mean renormalization group. This simple and well-understood tool can be employed and used advantageously in analyzing such a space. In fact, the present formula $d_{c}^{(4)}=(1 / \phi)^{3}$ reflects already some remarkably self-similar behaviour such as [3]

$$
1 /\left[d_{c}^{(4)}-4\right]=d_{c}^{(4)}
$$

which is of course in our context particularly suggestive.

Now, with $d_{c}^{(0)}=\phi$ we can say even more than just that $d_{c}^{(4)}=(1 / \phi)^{3}$. This is because $d_{c}^{(0)}=\phi$ is precisely the only Cantor set for which the average dimension $\langle d\rangle$ of an infinite dimensional set as defined in ref. [3] is also exactly equal to $(1 / \phi)^{3}$. In other words, the centre of an infinite dimensional random Cantor set is a four-dimensional Cantor set with average Hausdorff dimension [3]

$$
\langle d\rangle=\left(1 / d_{c}^{(0)}\right)^{3}=(1 / \phi)^{3} .
$$

A Cantor space or even a Peano-Hilbert space such as that used by Ord [7] may seem a little implausible and artificial, but is this really more artificial than the deadly flat space of our experience? Besides, if the sunflower is arranged according to the Golden Mean, to mention only one well-known example, why should not micro space have it as a Hausdorff dimension?

To question the very nature of the geometry of space and time is not as old as quantum mechanics-it is even older. However, only with the quarrel around the Copenhagen interpretation did such questions start to surface among eminent theoretical physicists such as Einstein, Pauli and Dirac [8].

However, it is interesting that no mathematical replacement for the smooth classical space-time was ever seriously formulated in a systematic way. By contrast, the topology of micro space-time, which received some extremely stimulating impulses from people like Wheeler and his school, have in the meantime evolved, due to some surprising, important new results in Knot theory, into impressive structure thanks to the work of Atiyah, Witten, Kaufman and their school. ${ }^{*}$ It is now known as quantum topology. To our knowledge there is no comparable work on the geometrical side except for that of Nottale and his school, of which we may mention the work of Le Méhaute on Maxwell equations.

In our opinion it is a very worthwhile enterprise to follow the idea of Cantorian space-time with all its mathematical and physical ramifications. The final version may well be a synthesis between the results of quantum topology, quantum geometry and maybe also Rössler's endophysics [9], which like Nottale's latest work makes extensive use of the ideas of Nelson's stochastic mechanics [10].

Acknowledgement-I am grateful to Otto Rössler for his extraordinary generosity and hospitality during an extremely pleasant and stimulating time which I spent with him and his group in beautiful Tübingen, and which prompted the writing of the present chain of thoughts.

\section{REFERENCES}

1. R. D. Mauldin and S. C. Williams, Random recursive construction, Trans. Am. Math. Soc. 295, 325-346 (1986).

\footnotetext{
* Similarities hetween quantum topology and superstrings as well as Penrose twistors have been investigated by Witten. This is important even though there are no immediate physical experiments to confirm the relevance of these ideas, starting from Kelvin's knotted vortex tubes of ether to Ichikawa and Wadati's loop solitons. It is even possible that the fractal approach to Einstein's dream of geometrizing physics is more relevant for scales above the Planck length where topology is still classical.
} 
2. M. S. El Naschie, Complex dynamics in 4D Peano-Hilbert space, Il Nuovo Cimento 107B, 589 (1992).

3. M. S. El Naschie, On dimensions of Cantor set related systems, Chaos, Solitons \& Fractals 3, 675-685 (1993).

4. L. Nottale, Int. J. mod. Phys. A4, 5047-5117. (1989).

5. M. S. El Naschie, Quantum mechanics and the possibility of a Cantorian spacetime, Chaos, Solitons \& Fractals 1, 485-487 (1992).

6. M. S. El Naschie, Statistical mechanics of multidimensional Cantor sets, Gödel theorem and quantum space-time, J. Franklin Inst. 330, 199-211 (1993).

7. G. N. Ord, J. Phys. A: Math. Gen. 16, 1869-1884 (1983).

8. M. S. El Naschie, Godel, Cantor and modern nonlinear dynamics, in First International Symposium on Gödel's Theorem (edited by Z. W. Wolkowski), pp. 95-106. World Scientific, Singapaore (1993).

9. O. E. Rössler, Intra observer chaos: hidden root of quantum mechanics, Chaos, Solitons \& Fractals 4 (to appear) (1994).

10. E. Nelson, Derivation of the Schrödinger equation from Newtonian mechanics, Phys. Rev. 150, 1079-1085 (1966). 